\title{
Unit Per Gram of Creatinine
}

National Cancer Institute

\section{Source}

National Cancer Institute. Unit Per Gram of Creatinine. NCI Thesaurus. Code C105523.

A unit of arbitrary substance concentration (biologic activity concentration) defined as the concentration of one unit per gram of creatinine. 